\title{
CARACTERIZAÇÃO DE LINHAGENS BACTERIANAS ISOLADAS DA BIODIVERSIDADE BRASILEIRA QUANTO À PRODUÇÃO DE BIOPOLÍMEROS
}

\section{FERNANDA MATIAS}

Tese apresentada ao Programa de Pós-Graduação Interunidades em Biotecnologia USP/Instituto Butantan/ IPT, para obtenção do Título de Doutor em Biotecnologia.

Área de concentração: Biotecnologia

Orientadora: Profa. Dra. Maria Filomena de Andrade Rodrigues

Co-orientador: Gabriel Padilla

São Paulo 


\section{RESUMO}

MATIAS, F. Caracterização de linhagens bacterianas isoladas da biodiversidade brasileira quanto à produção de biopolímeros. Tese (Doutorado em Biotecnologia) Instituto de Ciências Biomédicas da Universidade de São Paulo, São Paulo, 2009.

O lixo urbano tem sido apontado como um dos maiores poluentes ambientais sendo que o resíduo plástico chega a representar $20 \%$ do volume do lixo doméstico. Como alternativa aos plásticos petroquímicos, produtos plásticos menos agressivos ao meio ambiente e mais biodegradáveis têm sido estudados, entre eles os polihidroxialcanoatos (PHA). Os PHA são poliésteres biodegradáveis acumulados como material de reserva por inúmeras bactérias e que possuem aplicabilidade comercial bastante abrangente. Bactérias pertencentes ao gênero Burkholderia e ao grupo dos actinomicetos foram estudadas e despertaram interesses acadêmicos e industriais para a produção de diferentes composições poliméricas. No Brasil os primeiros estudos foram feitos na década de 1990, sendo identificadas novas linhagens bacterianas capazes de produzir diferentes PHA. Em trabalho prévio, 53 novas linhagens bacterianas produtoras de biopolímeros utilizando glicose ou glicose e caseína como fontes de carbono foram isoladas do solo do Rio Grande do Sul. Das 53 linhagens iniciais, 49 foram avaliadas neste trabalho quanto aos polímeros produzidos em diferentes fontes de carbono. Para selecionar as linhagens mais promissoras, os ensaios foram divididos em três fases principais: utilização do corante vermelho do Nilo, utilização do corante azul do Nilo e análise em cromatografia a gás dos possíveis polímeros produzidos. Verificando-se maior quantidade de fontes de carbono utilizadas, maior quantidade de produção de biopolímeros em função da quantidade de fontes de carbono utilizadas e variedade de polímeros produzidos, quatro linhagens foram selecionadas. Destas, duas linhagens foram identificadas como pertencentes ao grupo dos actinomicetos e duas foram identificadas como pertencentes ao gênero Burkholderia. As linhagens I29B (Burkholderia sp.) e Ind10J (Streptoallosporangium megapotamicus, proposta de gênero novo e espécie nova) foram avaliadas quanto à produção de um novo polímero composto por polihidroxibutirato (PHB) e polihidroxidodecanoato (PHDd) a partir de fontes de carbono não relacionadas. $A$ linhagem CC1J (Streptoalloteichus megapotamicus, proposta de nova espécie) demonstrou bons resultados na hidrólise de resíduos agroindustriais e produção de 
biopolímeros. As linhagens I29B e SA3J (Burkholderia cepacia) apresentaram amplificação do gene PHA sintase (phaC) de classe I, sendo que a linhagem SA3J também apresentou amplificação do gene phaC2 de classe II. A linhagem CC1J apresentou ambos os fragmentos do gene phaC de classe II, phaC1 e phaC2, sendo este um dado inédito. As linhagens Ind10J, CC1J e I29B foram analisadas em microscopia eletrônica de transmissão e varredura.

Palavras-chave: Actinomicetos, Actinobactérias, Burkholderia, PHA, PHB, PHDd, Resíduos industriais. 


\section{ABSTRACT}

MATIAS, F. Characterization of Brazilian biodiversity isolated bacterial strains on the production of biopolymer. Ph. D. thesis (Biotechnology) - Biomedical Sciences Institute of São Paulo University, São Paulo, 2009.

The urban waste has been identified as a major environmental pollutants and the plastic residue reaches $20 \%$ of the volume of household waste. As an alternative to petrochemical plastics, plastic products less harmful to the environment and more biodegradable have been studied, such as polyhydroxyalkanoates (PHA). PHA are biodegradable polyesters accumulated as carbon source reserve by many bacteria and have commercial applicability quite comprehensive. Bacteria belonging to the genus Burkholderia and to the group of actinomycetes have been studied and attracted academic and industrial interest for the production of different polymer compositions. In Brazil, first studies were done in the 1990s, being identified new bacterial strains capable to produce different PHA. In previous work, 53 new PHA-bacterial strains producers using glucose or glucose and casein as carbon sources were isolated from soil of Rio Grande do Sul. From 53 initial strains, 49 were evaluated in this work on the production of polymers in different carbon sources. To select the most promising strains, tests were divided into three main phases: use of the Nile red dye, use of Nile blue dye and analysis by gas chromatography of the possible polymers produced. Analyzing the greater amount of carbon sources used, greater amount of production of biopolymers by the quantity of carbon sources used and the variety of polymers produced, four strains were selected. Of these, two strains were identified as belonging to the group of actinomycetes and two were identified as belonging to the genus Burkholderia. Strains I29B (Burkholderia sp.) and Ind10J (Streptoallosporangium megapotamicus, proposal of new genus and new specie) were evaluated for production of a new polymer composed of polyhydroxybutyrate (PHB) and polyhydroxydodecanoate (PHDd) from unrelated carbon sources. The strain CC1J (Streptoalloteichus megapotamicus, proposal of new specie) showed good results in the hydrolysis of agroindustrial waste and production of biopolymers. Strains I29B and SA3J (Burkholderia cepacia) showed amplification of the PHA synthase gene ( $p h a C$ ) class I, and the strain SA3J also showed amplification of the gene phaC2 class II. The strain CC1J showed both gene fragments of class II phaC, 
phaC1 and phaC2, being this unprecedented. Strains Ind10J, CC1J and I29B were analyzed by transmission and scanning electron microscopy.

Key words: Actinomycetes, Actinobacteria, Burkholderia, PHB, PHA, PHDd, Industrial waste. 


\section{INTRODUÇÃO}

Em trabalho anterior, foram isoladas de diferentes solos do estado do Rio Grande do Sul 53 linhagens de actinomicetos produtoras de PHA a partir de glicose. Destas, 34 linhagens foram capazes de acumular PHB, sendo que sete linhagens acumularam entre $40 \%$ e $50 \%$. Algumas bactérias demonstraram capacidade de produzir outros polímeros em baixa quantidade. Os actinomicetos são bactérias abundantes no solo e que possuem uma variedade muito grande de enzimas extracelulares capazes de degradar os mais diversos substratos. Isso se torna interessante do ponto de vista industrial de produção de PHA já que podem utilizar como fontes de carbono resíduos industriais. Do ponto de vista de produção de PHA, são bactérias pouco exploradas para a produção de biopolímeros o que torna este trabalho bastante promissor quanto a caracterização de novos genes envolvidos no biossíntese de PHA. Além disso, são bactérias que crescem em diversas fontes de carbono de baixo custo, como rejeitos industriais, o que pode favorecer a produção de bioplástico em escala industrial a baixo custo. A fim de caracterizar os possíveis polímeros produzidos, assim como selecionar as bactérias mais interessantes, diferentes fontes de carbono de baixo custo foram utilizadas como: celulose, amido, lactose, glicerina, pectina, entre outros. Após a seleção das melhores linhagens foi feito o cultivo em resíduos industriais como bagaço de maçã e de laranja. A produção de biopolímeros a partir de resíduos é interessante por duas razões: utilização de matéria prima de baixo custo e redução de poluentes ambientais. A classificação das bactérias e busca dos genes envolvidos na biossíntese dos polímeros foi o próximo passo. 


\section{CONCLUSÕES}

Através deste trabalho pode-se concluir que:

- A bioprospecção foi eficiente em localizar bactérias produtoras de polímeros de cadeias curtas e médias e capazes de utilizar um grande número de fontes de carbono de baixo custo e não relacionadas aos polímeros produzidos.

- A caseína influi no crescimento e na produção dos polímeros pelas bactérias descritas neste trabalho;

- Quatro linhagens se destacaram quanto à utilização e produção de polímeros. De acordo com a análise taxonômica verificou-se que 2 linhagens não faziam parte do grupo das actinobactérias, mas sim ao gênero Burkholderia e ainda do genótipo Genomovar: Burkholderia sp. SA3J e Burkholderia sp. I29B. As outras duas linhagens pertenciam ao grupo das actinobactérias, sendo uma considerada gênero novo, espécie nova, Streptallosporangium megapotamicus Ind10J, e a outra espécie nova, Streptoalloteichus megapotamicus CC1J.

- As linhagens selecionadas foram capazes de produzir scl-PHA e mcl-PHA utilizando diversas fontes de carbono. A bactéria Streptoaleuriosporangium megapotamicus Ind10J produziu PHB, PHDd e PHTd a partir de pectina/citrato de sódio, enquanto a bactéria Burkholderia sp. I29B foi capaz de produzir PHB, PHV e PHDd a partir de glicose e caseína e glicose/sacarose e caseína. Já a bactéria Streptoalloteichus megapotamicus CC1J demonstrou bom crescimento nos resíduos industriais de laranja e maçã além de capacidade de hidrolisar celulose e hemicelulose produzindo PHB e PHDd a aprtir destes substratos.

- Com relação aos genes de biossíntese verificou-se que as bactérias Burkholderia sp. SA3J e Burkholderia sp. I29B amplificaram genes phaC de classe I, sendo que a bactéria Burkholderia sp. SA3J também amplificou phaC2 de classe II. A bactéria Streptoalloteichus megapotamicus CC1J 
amplificou ambas phaC1 e phaC2 do tipo II, podendo ainda apresentar mais PHA sintases, de acordo com resultados obtidos em experimentos de hibridação e amplificação genética. A bactéria Streptallosporangium megapotamicus. Ind10J não amplificou PHA sintases com os iniciadores utilizados. 


\section{REFERÊNCIAS BIBLIOGRÁFICAS*}

AMBIENTE BRASIL. Reciclagem; Plásticos; Reciclagem de PET no Brasil. Disponível em: < http://www.ambientebrasil.com.br>. Acesso em: 14 out. 2008

ALBERTS, B.; BRAY, D.; LEWIS, J.; RAFF, M.; ROBERTS, K.; WATSON, J. D. A Célula. 3 ed. Porto Alegre: Artes Médicas, 1997. 1294 p.

ALVAREZ, H. M. Relationship between B-oxidation pathway and the hydrocarbon-degrading profile in actinomycetes bacteria. Int. Biodeterior. Biodegrad., v.52, p. 35-42, 2003.

ALVAREZ, H. M.; MAYER, F.; FABRITIUS, D.; STEINBÜCHEL, A. Formation of introcytoplasmic lipid inclusions by Rhodococcus opacus strain PD630. Arch. Microbiol., v. 165, p. 377-386, 1996.

ALVAREZ, H. M.; STEINBÜCHEL, A. Triacylglycerols in prokaryotic microorganisms. Appl. Microbiol. Biotechnol., v. 60, p. 367-376, 2002.

AMERICAN CHEMISTRY. American Plastics Council. Available from: <http://www.americanplasticscouncil.org/benefits>. Acesso em: 20 Fev. 2003.

ANDERSON, A. A.; DAWES, E. A. Occurrence, metabolism, metabolic role, and industrial uses of bacterial polyhydroxyalkanoates. Microbiol. Rev., v.54, p. 450-472, 1990.

ASRAR, J.; VALENTIN, H. E.; BERGER, P. A.; TRAN, M.; PADGETTE, S. R.; GARBOW, J. R. Biosynthesis and properties of poly(3-hydroxybutyrate-co-3-hydroxyhexanoate) polymers. Biomacromol., v. 3, p. 1006-1012, 2002.

AUSUBEL, E.; BRENT, R.; KINGSTON, R. E.; MOORE, D. D.; SEIDMAN, J. G.; SMITH, J. A.; STUHL, K. Short protocols in molecular biology. $5^{\text {th }}$ ed. New York: John Wiley, 2002.

BARABÁS, G.; VARGHA, G.; SZABÓ I. M.; PENYIGE, A.; DAMJANOVICH, S.; SZÖLLÖSI, J.; MATKÓ, J.; HIRANO, T.; MÁTYUS, A.; SZABÓ, I. N-Alkane uptake and utilisation by Streptomyces strains. Ant. Van Leeuw., v. 79, n. 3-4, p. 269-76, 2001.

BARBUZZI, T.; GIUFFRIDA, M.; IMPALLOMENI, G.; CARNAZZA, S. Microbial synthesis of poly(3-hydroxyalkanoates) by Pseudomonas aeruginosa from fatty acids: identification of higher monomer units and structural characterization. Biomacromol., v. 5, p. 2469-2478, 2004. 
BIRNBORIM, H. C.; DOLY, J. A rapid extraction procedure for screening recombinant plasmid DNA. Nucleic Acids Res., v.7, p.1513-1523, 1979.

BIOPOL. PHB accumulation in Micro-organisms. Available from: <http://members.rediff.com/jogsn/BP7.htm>. Acesso em: 6 Nov. 2003.

BLAST- Basic Local Alignment Search Tool. Available from: $<$ http://blast.ncbi.nlm.nih.gov/Blast.cgi>.

BONATTO, D. Isolamento e caracterização de novas linhagens eubacterianas produtoras de ácidos poli(hidroxialcanóicos). 96f. Dissertação (Mestrado em Biotecnologia) - Centro de Biotecnologia, Universidade Federal do Rio Grande do Sul, Porto Alegre, 2000.

BONATTO, D.; MATIAS, F.; LISBÔA, M. P.; BOGDAWA, H. M.; HENRIQUES, J. A. P. Production of short side chain-poly(hydroxyalkanoate) by a newly isolated Ralstonia picketti strain. World J. Microbiol. Biotechnol., v. 20, p. 395-403, 2004.

BRAUNEGG, G.; SONNELEITNER, B.; LAFFERTY, R. M. A rapid gas chromatographic method for the determination of poly-b-hydroxybutiric acid in microbial biomass. Eur. J. Appl. Microbiol. Biotechnol., v. 6, p. 29-37, 1978.

BROCK, T. D.; SMITH, D. W.; MADIGAN, M. T. Biology of Microrganisms. London: Prentice Hall, 1998.

BYROM, D. Polymer synthesis by microorganisms: technology and economics. TIBTECH, v.5, p. 246-250, 1987.

CALABIA, B. P.; TOKIWA, Y. Microbial degradation of poly(ß-3-hydroxybutyrate) by a new thermophilic Streptomyces isolate. Biotechnol. Lett., v. 26, p. 15-19, 2004.

CHEN, G. Q.; WU, Q. The application of polyhydroxyalkanoates as tissue engineering materials. Biomaterials, v. 26 p. 6565-6578, 2005.

CHUN, J. Computer-assisted classification and identification of actinomycetes. $\mathrm{PhD}$ Thesis - University of Newcastle upon Tyne (UK), 1995.

D’ALMEIDA, M. L. O., VILHENA, A. Lixo municipal: Manual de gerenciamento integrado. 2 ed. São Paulo: IPT/CEMPRE, 2000. 370 p.

DUNCAN, D. K. The Society of the Plastic Industry. Available from: <http://www.plasticindustry.org>. Acesso em: 16 Maio 2000. 
ENSIGN, J. C. Formation, properties, and germination of actinomycetes spores. Ann. Rev. Microbiol., v.32, p.185-219, 1978.

ESPACENET. Available from: <http://ep.espacenet.com/>.

FERGUSON, D. A. J.: e CUMMINS, C. S. Nutritional Requirements of Anaerobic Coryneforms. J. Bacteriol., v. 135, n. 3, p. 858-867, 1978.

FIECHTER, A. Plastics from Bacteria and for Bacteria: Poly (B-Hydroxyalkanoates) as Natural, Biocompatible, and Biodegradable Polyesters. New York: Springer-Verlag, 1990, p. $77-93$.

FONSECA, G. G. Produção de polihidroxialcanoatos por Escherichi coli recombinante. 178 f. Dissertação (Mestrado em Engenharia de Alimentos) - Engenharia de Alimentos, Universidade Federal de Santa Catarina, Florianópolis, 2003.

FULLER, R. C. Microbial inclusions with special reference to PHA inclusions and intracellular boundary envelopes. Int. J. Biol. Macromol., v.25, p. 21-29, 1999.

GOMEZ, J.G.C.; RODRIGUES, M.F.A.; ALLI, R.C.P. TORRES, B. B.; BUENO NETTO, C. L.; OLIVEIRA, M. S.; DA SILVA, L. F. Evaluation of soil Gram-negative bacteria yielding polyhydroxyalkanoic acids from carbohydrates and propionic acid. Appl. Microbiol. Biotechnol., v.45, p. 785-791, 1996.

GORENFLO, V.; SCHMACK, G.; VOGEL, R.; STEINBUCHEL, A. Development of a Process for the Biotechnological Large-Scale Production of 4-Hydroxyvalerate-Containing Polyesters and Characterization of Their Physical and Mechanical Properties. Biomacromol., v. 2, p. 45-57, 2001.

GREENSPAN, P.; MAYER, E.P.; FOWLER, S.D. Nile red: a selective fluorescent stain for intracellular lipid droplets. J. Cell Biol., v. 100, 965-973, 1985.

GRUBELNIK, A.; WIESLI, L.; FURRER, P.; RENTSCHR, D.; HANYZ, R.; MEYER, V.R. A simple HPLC-MS method for the quantitative determination of the composition of bacterial medium chain-length polyhydroxyalkanoates. J. Sep. Sci., v.31, 1739 - 1744, 2008.

HALL, T.A. BioEdit: a user-friendly biological sequence alignment editor and analysis program for Windows 95/98/NT. Nucl. Acids. Symp., v. 41, p. 95-98, 1999.

HALL, B., BALDWIN, J., RHIE, H.G., DENNIS, D.. Cloning of the Nocardia corallina polyhydroxyalkanoate synthase gene and production of poly-(3-hydroxybutyrate-co3-hydroxyhexanoate) and poly-(3-hydroxyvalerate-co-3-hydroxyheptanoate). Can. J. Microbiol., v. 44, p. 687-691, 1998. 
HANG, X.; ZHANG, G.; WANG, G.; ZHAO, X. CHEN, G. PCR cloning of polyhydroxyalkanoates biosinthesis genes from Burkholderia caryophylli and their functional expression in recombinant Escherichia coli. FEMS Microbiol. Lett., v. 210, p. 49-54, 2002.

HANKERMEYER, C. R.; TJEERDEMA, R. S. Polyhydroxybutyrate: plastic made and degraded by microorganisms. Rev. Environ. Contam. Toxicol., v.159, p. 1-24, 1999.

HAYWOOD, G. W.; ANDERSON, A. J.; CHU, L.; DAWES, E. A. Accumulation of polyhydroxyalkanoates by bacteria and the substrate specificities of the biosynthetic enzymes. Biochem. Soc. Trans., v.60, p.1046-1047, 1988a.

HAYWOOD, G. W.; ANDERSON, A. J.; CHU, L.; DAWES, E. A. Characterization of two 3ketothiolases possessing differing substrate specificities in the polyhydroxyalkanoate synthesizing organism Alcaligenes eutrophus. FEMS Microbiol. Lett., v.52, 1-2, p.91-96, jul. 1988b.

HAYWOOD, G. W.; ANDERSON, A. J.; WILLIAMS, D. R.; DAWES, E. A.; EWING, D. F. Accumulation of a poly(hydroxyalkanoate) copolymer containing primarily 3-hydroxyvalerate from simple carbohydrate substrates by Rhodococcus sp. NCIMB 40126. Int. J. Biol Macromol., v.13, 2, p. 83-88, 1991.

HOFFMANN, N.; AMARA, A. A.; BEERMANN, B. B; QI, Q.; HINZ, H. J; REHM, B. H. A. Biochemical characterization of the Pseudomonas putida 3-hydroxyacil ACP:CoA transacylase, wich diverts intermediates of fatty acids de novo biosynthesis. J. Biol. Chem., v.277, n. 45, p. 42926-42936, 2002.

HOLT, J.; KRIEG, N. R.; SNEATH, P.H.A. Bergey's manual of determinative bacteriology. 9 ed. Baltimore: Williams \& Wilkins, 1994.

HUISMAN, G. W.; WONINK, E.; MEIMA, R.; KATZEMIER, B.; TERPSTRA, P.; WITHOLT, B. Metabolism of poly(3-hydroxyalcanoates) by Pseudomonas oleovorans: identification and sequences of genes and function of the encoded proteins in the synthesis and degradation of PHA. J. Biol. Chem., v.266, n. 4, p. 2191-2198, 1991.

INDUSTRY CANADA. Canadian Plastics Industry. Available from: $<$ http://www.ic.gc.ca/epic/site/plastics-plastiques.nsf/en/pl00312e.html>. Acesso em: 14 Out. 2008.

JENDROSSEK, D.; HANDRICK, R. Microbial degradation of polyhydroxyalkanoates. Annu. Rev. Microbiol.. v. 56, p. 403-32, 2002.

JONES, K. L. Fresh isolates of actinomycetes in wich the presence of sporogenous aerial mycelia is a fluctuating characteristic. J. Bacteriol., v. 57, p. 141-145, 1949. 
KAMIYA, N.; YAMAMOTO,Y.; INOUE,Y.; CHUJO, R.; DOI,Y. Microstructure of bacterially synthesized poly(3-hydroxybutyrate-co-3-hydroxyvalerate). Macromol., v. 22, p. 1676-1682, 1989.

KANNAN, L. V.; REHACEK, Z. Formation of poly- $\beta$-hydroxybutyrate by Actinomycetes. Ind. J. Biochem., v.7, p. 126-129, 1970.

KIESER, T.; BIBB, M. J.; BUTTNER, M. J.; CHATER, K. F.; HOPWOOD, D.A. Practical Streptomyces Genetics. Norwich, England: The John Innes Foundation, 2000.

KIM ,H. J.; KIM, D. Y.; Nam, J. S.; BAE, K. S.; RHEE, Y. H. Characterization of an extracellular medium-chain-length poly(3-hydroxyalkanoate) depolymerase from Streptomyces sp. KJ-72. Ant. Leeuw., v. 83, p. 183-189, 2003.

KLINGBEIL, B.; KROPPENSTEDT, R. M.; JENDROSSEK, D. Taxonomic identification of Streptomyces exfoliatus $\mathrm{KIO}$ and characterization of its poly(3-hydroxybutyrate) depolymerase gene. FEMS Microbiol. Lett., v. 142, p. 215-221, 1996.

KULKARNI, M.; CHAUDHARI, A. Microbial remediation of nitro-aromatic compounds: an overview. J. Environ. Manag., v.85, n. 2, p. 496-512, 2007.

LANE, D. J. 16S/23S rRNA sequencing. In: E. STACKEBRANDT; M. GOODFELLOW (Ed.). Nucleic acid techniques in bacterial systematics. New York: John Wiley and Sons, 1991. p. 115-175.

LEMOIGNE, M. Produits de déshydration et de polymérisation de l'acide $\beta$-oxybutyrique. Bull. Soc. Chim. Biol., Paris, p. 770-782, 1926.

LEMOIGNE, M.; ROUKHELMAN, N. Ann. Ferment., v.5, p. 527, 1940.

LIU, W.; HANADA, S.; MARSH, T. L.; KAMAGATA, Y.; NAKAMURA, K. Kineosphaera limosa gen. nov., sp. nov., a novel Gram-positive polyhydroxyalkanoate-accumulating coccus isolated from activated sludge. Int. J. System. Evol. Microbiol., v. 52, p. 1845-1849, 2002.

MADISON, L. L.; HUISMAN, G. W. Metabolic engineering of poly(3-hydroxyalkanoates): from DNA to plastic. Microbiol. Mol. Biol. Rev., v. 63, n.1, p. 21-53, 1999.

MAHENTHIRALINGAM, E.; BISCHOF, J.; BYRNE, S. K.; RADOMSKI, C.; DAVIES, J. E.; AV-GAY, Y.; VANDAMME, P. DNA-based diagnostic approaches for identification of Burkholderia cepacia complex, Burkholderia vietnamiensis, Burkholderia multivorans, Burkholderia stabilis, and Burkholderia cepacia genomovars I and III. J. Clin. Microbiol., v. 38, p. 3165-3173, 2000.

MANNA, A.; BANERJEE, R.; PAUL A. K. Accumulation of poly (3-hydroxybutyric acid) by some soil Streptomyces. Curr. Opin. Microbiol., v.39, n. 3, p. 153-8, 1999. 
MATIAS, F. Isolamento e caracterização de novas linhagens de actinomicetos produtoras de PHAs de solos do Rio Grande do Sul. 74f. Monografia (Bacharelado em Biotecnologia) - Centro de Biotecnologia, Universidade Federal do Rio Grande do Sul, Porto Alegre, 2003.

MATIAS, F.; BONATTO, D.; PADILLA, G.; RODRIGUES, M. F. A.; HENRIQUES, J. A. P. Polyhydroxyalkanoates production by actinobacteria isolated from soil. Can. J. Microbiol., in press, 2009.

MIS OPERATING MANUAL - Microbial Identification System Sherlock. MIDI Inc., Newark, USA, 2001.

MONCIARDINI, P., et al., New PCR primers for the selective amplification of 16S rDNA from different groups of actinomycetes. FEMS Microbiol. Ecol., v.1414, p.1-11, 2002.

MONTEIRO, C. Como funciona a reciclagem de plástico. Disponível em: <http://ambiente.hsw.uol.com.br/reciclagem-plastico.htm>. Acesso em 14 out. 2008.

OSTLE, A. G., HOLT, J. G. Nile blue A as a Fluorescent Stain for Poly- $\beta$-hydroxybutyrate. Appl. Env. Microbiol., v.1, n.44, p.238-241, 1982.

PEFEROEN, M.; HUYBRECHTS, R.; LOOF, A. Vaccum-blotting: a new simple and efficient transfer of proteins from sodium dodecylsulfate polyacrylamide gels to nitrocellulose. FEBS Lett., v. 145, p.369-372, 1982.

PERRONE, C. Biodegradabilidad: Siempre de moda. Tec. Plastico, v.49, p.14-21, 1991.

PIEPER, U. Biosynthese eines copolymers aus 3-hydroxybuttersaüre und 3hydroxyvaleriansaüre in Rhodococcus rubber NCIMB 40126: physiologische, molecular - genetische und biochemische Untersuchungen. Tese (Doutorado em Microbiologia) Universidade de Göttingen, Alemanha, 1993.

PIEPER, D. E.; SEEGER, M. Bacterial metabolism of polychlorinated biphenyls. J. Mol. Microbiol. Biotechnol., v.15 n. 2-3, p.121-38, 2008.

PIEPER, U.; STEINBÜCHEL, A. Identification, cloning and sequence analysis of the poly(3hydroxyalkanoic acid) synthase gene of the gram-positive bacterium Rhodococcus rubber. FEMS Microbiol. Lett., v. 96, p. 73-80, 1992.

PHILIPS, S.; KESHAVARZ, T.; ROY, I. Polyhydroxyalkanoates: biodegradable polymers with a range of applications. J. Chem. Technol. Biotechnol., p. 82, n. 3, p. 233-247, 2007.

PLASTICS EUROPE. Association of Plastics Manufacturers. Available from: <http://www.plasticseurope.org/Content/Default.asp?>. Acesso em: 14 Out. 2008. 
PLASTIVIDA. Plásticos em harmonia com a vida. Disponível em: <http://www.plastivida.org.br/os_plasticos/oquesao.htm>. Acesso em 14 out. 2008.

POIRIER, Y.; NAWRATH, C.; SOMERVILLE, C. Production of polyhydroxyalkanoates, a family of biodegradable plastics and elastomers, in bacteria and plants. Bio/technology, $\mathrm{v}$. 13, p. 142-150, 1995.

POOL, R. In search of the plastic potato. Science, v. 245, p. 1187-1189, 1989.

RAMACHANDER, T. V. N.; RAWAL, S. K. PHB synthyase from Streptomyces aureofaciens NRRL 2209. FEMS Microbiol. Lett., v. 242, p. 13-18, 2005.

RAMACHANDER, T. V. N.; ROHINI, D.; BELHEKAR, A.; RAWAL, S. K. Synthesis of PHB by recombinant $E$. coli harboring an approximately $5 \mathrm{~Kb}$ genomic DNA fragment from Streptomyces aureofaciens NRRL 2209. Int. J. Biol. Macromol., v.31, p. 63-69, 2002.

RAINEY, F.A.; WARD-RAINEY, N.; KROPPENSTEDT, R.M.; STACK-BRANDT, E. The genus Nocardiopsis represents a philogenetically coherent taxon and a distinct actinomycete lineage: proposal of Nocardiopsaceae fam. nov. Appl. Environ. Microbiol., v. 56, p. 20932098, 1990.

REHM, B. H. A. Polyester synthases: natural catalysts for plastics. Biochem. J., v. 376, p. 15-33, 2003.

REHM, B. H. A.; STEINBÜCHEL, A. Biochemical and genetic analysis of PHA synthases and other proteins required for PHA synthesis. Int. J. Biol. Macromol., v.25, p. 3-19, 1999.

RDP - Ribosomal Database Project. Available from: <http://rdp.cme.msu.edu/>.

RICHERT, K.; BRAMBILLA, E.; STACKEBRANDT, E. Development of PCR primers specific for the amplification and direct sequencing of gyrB genes from microbacteria, order Actinomycetales. J. Microbiol. Methods, v.60, n. 1, p.115-23, 2005.

RICHERT, K.; BRAMBILLA, E.; STACKEBRANDT, E. The phylogenetic significance of peptidoglycan types: Molecular analysis of the genera Microbacterium and Aureobacterium based upon sequence comparison of gyrB, rpoB, recA and ppk and 16SrRNA genes. Syst. Appl. Microbiol., v. 30, n. 2, p. 102-108, 2007.

RIIS, V.; MAI, W. Gas chromatography determination of poly-ß-hydroxybutyric acid in microbial biomass after hydrocloric acid propanolisis. J. Chromatogr., v. 445, p. 285-289, 1988.

RODRIGUES, M. F. A. Isolamento e caracterização de bactérias quanto à produção de polihidroxialcanoatos (PHAs) e clonagem dos genes de biossíntese de PHA de 
Burkholderia sp. 151 f. Dissertação (Mestrado em Microbiologia) - Instituto de Ciências Biomédicas, Universidade de São Paulo, São Paulo, 1995.

RODRIGUES, M. F. A. Estudo da biossíntese de polihidroxialcanoatos (PHAs) em Burkholderia cepacia linhagem IPT64. 130f. Tese (Doutorado em Microbiologia) - Instituto de Ciências Biomédicas, Universidade de São Paulo, São Paulo, 2000.

RUEDA, B.; MIGUÉLEZ, E. M.; HARDISSON, C. MANZANAL, M. B. Changes in glycogen and trehalose content of Streptomyces brasiliensis hyphae during growth in liquid cultures under sporulating and non-sporulating conditions. FEMS Microbiol. Lett., v.194, p. 181-185, 2001.

SAMBROOK J.; RUSSELL D.W. Molecular cloning. A laboratory manual. $3^{\text {rd }}$ ed. New York: Cold Spring Harbor Laboratory Press, 2001.

SCHIMIDELL, W.; VITORATTO, E. O desafio do lixo urbano. Rev. Politécnica, v.204/205, p. 40-42, 1992.

SCHLEGEL, H. G.; LAFFERTY, R.; KRAUSS, I. The isolation of mutants not accumulating poly- $\beta$-hydroxybutyric acid. Arch. Mikrobiol., v.71, p. 283-294, 1970.

SCIENCE EDUCATION - Bacterial Plastic - Available from: $<$ http://www.bact.wisc.edu:81/ScienceEd/discuss/msgReader\$10>. Acesso em: 6 Nov. 2003.

SERAFIM, L. S. ; LEMOS, P. C.; TORRES, C.; REIS, M. A. M.; RAMOS, A. M. The Influence of Process Parameters on the Characteristics of Polyhydroxyalkanoates Produced by Mixed Cultures. Macromol. Biosci., v. 8, p. 355-366, 2008.

SHEU, D.; WANG, Y.; LEE, C. Rapid detection of polyhydroxyalkanoate accumulating bacteria isolated from the environment by colony PCR. Microbiology, v. 146, p. 2019-2025, 2000.

SHIRLING, E. B.; GOTTLIEB, D. Methods for characterization of Streptomyces species. Int. J. Syst. Bacteriol., v.16, p.313-340, 1966.

SIRESP. Sindicato da Indústria de Resinas Sintéticas no Estado de São Paulo - Matéria Prima para os Plásticos do Brasil. Disponível em: <http://www.siresp.org.br>. Acesso em: 24 set. 2003.

SOLAIMAN, D. K.Y. Polymerase-chain-reaction-based detection of individual polyhydroxyalkanoate synthase phaC1 and phaC2 genes. Biotechnol. Lett., v. 24, p. 245250, 2002. 
SOLAIMAN, D.K.Y.; ASHBY R.D. Rapid genetic characterization of poly(hydroxyalkanoate) synthase and its applications. Biomacromolecules, v. 6, p. 532-537, 2005.

SOLAIMAN, D. K. Y.; ASHBY, R. D.; FOGLIA, T. A. Rapid and specific identification of medium-chain-length polyhydroxyalkanoate synthase gene by polymerase chain reaction.

Appl. Microbiol. Biotechnol., v. 53, p. 690-694, 2000.

SOLAIMAN, D. K.Y.; ASHBY, R. D.; HOTCHKISS JR, A. T.; FOGLIA, T. A. Biosynthesis of medium-chain-length poly(hydroxyalkanoates) from soy molasses. Biotechnol. Lett., v. 28, p. 157-162, 2006.

SPIEKERMANN, P.; REHM, B. H.; KALSCHEUER, R.; BAUMEISTER, D.; STEINBÜCHEL, A. A sensitive, viable-colony staining method using Nile Red for direct screening of bacteria that accumulate polyhydroxyalkanoic acids and other lipid storage compounds. Arch. Microbiol., v.171, p. 73-80, 1999.

STACKEBRANDT, E.; RAINEY, F. A.; WARD-RAINEY, N. L. Proposal for a new Hierarchic classification system, Actinobacteria classis nov. Int. J. Syst. Bacteriol., v.47, p.479-491, 1997.

STATISCS NORWAY. Waste accounts for plastic, 1986-1997. Available from: <http://www.sbs.no.english/subjects/01/05/40/avfregnplast_en/main.html>. Acesso em: 20 Fev. 2003.

STEIN, R. S. Polymer recycling: opportunities and limitations. Proc. Natl. Acad. Sci., v.89, p. 835-838, 1992.

STEINBÜCHEL, A. PHB and other Polyhydroxyalkanoic acids. In: REHM, H.J.; REED, G. ROEHR, M. (Ed.). Products of primary metabolism. Weinheim: Willey VCH, 1996. p.405464.

STEINBÜCHEL, A.; HUSTEDE, E.; LIEBERGESELL, M.; PIEPER, U.; TIMM, A.; VALENTIN, $H$. E. Molecular basis for biosyntesis and accumulation of polyhydroxyalcanoic acids in bacteria. FEMS Microbiol. Rev., v.103, p. 217-230, 1992.

STEINBÜCHEL, A.; VALENTIN, H.E. Diversity of bacterial polyhydroxyalkanoic acids. FEMS Microbiol. Lett., v.128, p. 219-228, 1995.

SUDESH, K.; ABE, H.; DOI, Y. Synthesis, structure and properties of polyhydroxyalkanoates: biological polyesters. Prog. Polym. Sci., v. 25, p. 1503- 1555, 2000.

TAMURA, K. Estimation of the number of nucleotide substitutions when there are strong transitition-transversion and G+C-content biases. Mol. Biol. Evol., v.9, p. 678-687, 1992. 
TAMURA K.; DUDLEY J.; NEI M.; KUMAR S. MEGA4: Molecular Evolutionary Genetics Analysis (MEGA) software version 4.0. Mol. Biol. Evol., v. 24, p. 1596-1599, (2007). (Publication PDF at http://www.kumarlab.net/publications)

TIAN, J.; HE, A; LAWRENCE, A. G.; LIU, P.; WATSON, N.; SINSKEY, A. J; STUBBE, J. Analysis of transient polyhydroxybutyrate production in Wautersia eutropha $\mathrm{H} 16$ by quantitative western analysis and transmission electron microscopy. J. Bacteriol., v.187, n.11, p. 3825-3832, 2005.

TOKIWA, Y.; CALABIA, B. P. Degradation of microbial polyesters. Biotechnol. Lett., v. 26, p. 1181-1189, 2004.

TSUGE, T.; WATANABE, S.; SHIMADA, D.; ABE, H.; DÓI, Y.; TAGUCHI, S. Combination ofN149S andD171G mutations in Aeromonas caviae polyhydroxyalkanoate synthase and impact on polyhydroxyalkanoate biosynthesis. FEMS Microbiol. Lett., v. 277, p. 217-222, 2007.

TUNG, L.H.; BUCKSER, S. The Effects of Molecular Weight on the Crystallinity of Polyethylene. J. Phys. Chem., v. 62, p. 1530-1534, 1958.

VAINBERG, S.; McCLAY, K.; MASUDA, H.; ROOT, D.; CONDEE, C.; ZYLSTRA, G.J.; STEFFAN, R.J. Biodegradation of Ether Pollutants by Pseudonocardia sp. Strain ENV478. Appl. Environ. Microbiol., v.72, p. 5218-5224, 2006.

VALAPPIL, S. P.; BOCCACCINI, A. R.; BUCKE, C.; ROY, I. Polyhydroxyalkanoates in Grampositive bacteria: insights from the genera Bacillus and Streptomyces. Ant. Leeuw., v. 91, p. 1-17, 2007.

VOET, D.; VOET, J.G. Biochemistry. New York: John Wiley \& Sons, 1990.

WANG, Q, G. M. GARRITY, J. M. TIEDJE, AND J. R. COLE. Naïve Bayesian classifier for rapid assignment of rRNA sequences into the new bacterial taxonomy. Appl. Environ. Microbiol., v. 73, 16, p.5261-5267. 2007

WILLIAMS, S. T.; GOODFELLOW, M.; ALDERSON, G.; WELLINGTON, E. M. H.; SNEATH, P. H. A.; SACKIN, J. Numerical classification of Streptomyces and related genera. J. Gen. Microbiol., v.129, p.1743-1813, 1983a.

WILLIAMS, S. T.; GOODFELLOW, M.; WELLINGTON, E. M. H.; VICKERS, J. C.; ALDERSON, G.; SNEATH, P. H. A.; SACKIN, J.; MORTIMER, A. M. A probability matrix for identification of some Streptomycetes. J. Gen. Microbiol., v.1815-1830, 1983b.

WILLIAMS, S. T.; SHARPE, M.E.; HOLT, J.G. Bergeys Manual of Systematic Bacteriology. Baltimore, USA: Williams \& Wilkins, 1989. 
WITHOLT, B.; KESSLER, B. Perspectives of medium chain length poly(hydroxyalkanoates), a versatile set of bacterial bioplastics. Curr. Opin. Biotechnol., v. 10, p. 279-285, 1999. 